\title{
Investigation of the Sensitivity of the Monte Carlo Solution for the Barker-Ferry Equation with Sequential and Parallel Pseudo-Random Number Generators ${ }^{\star}$
}

\author{
T.V. Gurov ${ }^{1}$ and P.A. Whitlock ${ }^{2}$ \\ 1 IPP - BAS, Acad. G. Bonchev st, bl. 25 A, \\ 1113 Sofia, Bulgaria, gurov@parallel.bas.bg \\ 2 Dep. of CIS, BC-CUNY, 2900 Bedford Ave, \\ Brooklyn, NY 11210, USA, whitlock@sci.brooklyn.cuny.edu
}

\begin{abstract}
In this work a set of several sequential and parallel pseudorandom number generators (prng's) are tested when we apply a MC approach for solving a quantum-kinetic equation derived from ultra-fast transport in semiconductos. The aim is an optimization of the MC solver for the equation which accounts for quantum effects in the electronphonon interaction. We consider so-called the Barker-Ferry (B-F) equation written for the case of zero electric field. The original formulation of this equation accounts for the action of the electric field during the process of collision.

The sensitivity of the MC solution for the electron energy distribution is investigated empirically, using prng's under consideration. The results obtained for the computational cost of the MC algorithm, the accuracy and the bias in the MC solution can be used to guide the choice of rng in the general case.
\end{abstract}

\section{The Quantum-Kinetic Equation}

The Barker-Ferry equation [1 can be utilized as a relevant physical model of the femtosecond relaxation of initially excited by a laser pulse electrons. For zero electrical field, the equation can be written in the following integral form 2]:

$$
\begin{aligned}
& f(\mathbf{k}, t)=\int_{0}^{t} d t^{\prime} \int_{0}^{t^{\prime}} d t^{\prime \prime} \int d^{3} \mathbf{k}^{\prime}\left\{S\left(\mathbf{k}^{\prime}, \mathbf{k}, t^{\prime}-t^{\prime \prime}\right) f\left(\mathbf{k}^{\prime}, t^{\prime \prime}\right)\right. \\
&\left.-S\left(\mathbf{k}, \mathbf{k}^{\prime}, t^{\prime}-t^{\prime \prime}\right) f\left(\mathbf{k}, t^{\prime \prime}\right)\right\}+\phi(\mathbf{k}), \\
& S\left(\mathbf{k}^{\prime}, \mathbf{k}, t^{\prime}-t^{\prime \prime}\right)=\frac{2 V}{(2 \pi)^{3} \hbar^{2}}\left|g_{\mathbf{k}^{\prime}-\mathbf{k}}\right|^{2} \exp \left(-\Gamma\left(\mathbf{k}^{\prime}, \mathbf{k}\right)\left(t^{\prime}-t^{\prime \prime}\right)\right) \\
& \times\left\{\left(n_{\mathbf{q}}+1\right) \cos \left(\Omega\left(\mathbf{k}^{\prime}, \mathbf{k}\right)\left(t^{\prime}-t^{\prime \prime}\right)\right)+n_{\mathbf{q}} \cos \left(\Omega\left(\mathbf{k}, \mathbf{k}^{\prime}\right)\left(t^{\prime}-t^{\prime \prime}\right)\right)\right\},
\end{aligned}
$$

\footnotetext{
* Supported by ONR Grant N00014-96-1-1-1057 and by Center of Excellence BIS-21 grant ICA1-2000-70016, as well as by the NSF of Bulgaria through grant number $\mathrm{I}-1201 / 02$.
} 
where $\mathbf{k}$ is the momentum, $f(\mathbf{k}, t)$ is the distribution function and $\phi(\mathbf{k})$ is the positive initial condition. In the kernel (2), $n_{\mathbf{q}}$ is the Bose function [2], $V$ is the volume and $\Omega\left(\mathbf{k}^{\prime}, \mathbf{k}\right)=\left(\varepsilon\left(\mathbf{k}^{\prime}\right)-\varepsilon(\mathbf{k})-\hbar \omega_{\mathbf{q}}\right) / \hbar$. The phonon energy is $\hbar \omega_{\mathbf{q}}$, which generally depends on $\mathbf{q}=\mathbf{k}^{\prime}-\mathbf{k}$, and $\varepsilon(\mathbf{k})=(\hbar \mathbf{k})^{2} / 2 m$ is the electron energy. The coupling $g_{\mathbf{k}^{\prime}-\mathbf{k}}=-i\left[\frac{2 \pi e^{2} \hbar \omega_{\mathbf{q}}}{V}\left(\frac{1}{\epsilon} \infty \frac{1}{\epsilon}_{s}\right) \frac{1}{\left(\mathbf{k}^{\prime}-\mathbf{k}\right)^{2}}\right]^{\frac{1}{2}}$ applies to the Fröhlich interaction, and $\left(\epsilon_{\infty}\right)$ and $\left(\epsilon_{s}\right)$ are the optical and static dielectric constants. The damping factor $\Gamma\left(\mathbf{k}^{\prime}, \mathbf{k}\right)=\Gamma\left(\mathbf{k}^{\prime}\right)+\Gamma(\mathbf{k})$ is related to the finite carrier lifetime for the scattering process: $\Gamma(\mathbf{k})=\int d^{3} \mathbf{k}^{\prime} \frac{V}{2^{3} \pi^{2} \hbar} \sum_{ \pm}\left\|g_{\mathbf{k}^{\prime}-\mathbf{k}}\right\|^{2} \delta\left(\varepsilon\left(\mathbf{k}^{\prime}\right)-\right.$ $\left.\varepsilon(\mathbf{k}) \pm \hbar \omega_{\mathbf{q}}\right)\left(n_{\mathbf{q}}+\frac{1}{2} \pm \frac{1}{2}\right)$. In spherical coordinates $(k, \theta, \varphi)$, with the $k_{z}^{\prime}$ axis oriented along $\mathbf{k}$ and zero lattice temperature $\left(n_{\mathbf{q}}=0\right)$, the equation (1) becomes one-dimensional with respect to the momentum integration [3]:

$$
\begin{gathered}
f(k, t)=\int_{0}^{t} d t^{\prime \prime} \int_{0}^{Q} d k^{\prime} K\left(k, k^{\prime}\right) \times \\
\times\left[K_{1}\left(k, k^{\prime}, t, t^{\prime \prime}\right) f\left(k^{\prime}, t^{\prime \prime}\right)+K_{2}\left(k, k^{\prime}, t, t^{\prime \prime}\right) f\left(k, t^{\prime \prime}\right)\right]+\phi(k), \\
K\left(k, k^{\prime}\right)=c_{1} \frac{k^{\prime}}{k} \ln \left(\frac{k+k^{\prime}}{\left|k-k^{\prime}\right|}\right), \\
K_{1}\left(k, k^{\prime}, t, t^{\prime \prime}\right)=-K_{2}\left(k^{\prime}, k, t, t^{\prime \prime}\right)=\frac{1}{\Omega_{k^{\prime}, k}^{2}+\Gamma_{k^{\prime}, k}^{2}}\left\{\Gamma_{k^{\prime}, k}+\right. \\
\left.+\exp \left(-\Gamma_{k^{\prime}, k}\left(t-t^{\prime \prime}\right)\right)\left[\Omega_{k^{\prime}, k} \sin \left(\Omega_{k^{\prime}, k}\left(t-t^{\prime \prime}\right)\right)-\Gamma_{k^{\prime}, k} \cos \left(\Omega_{k^{\prime}, k}\left(t-t^{\prime \prime}\right)\right)\right]\right\}
\end{gathered}
$$

and $c_{1}=e^{2} \omega\left|\frac{1}{\epsilon_{\infty}}-\frac{1}{\epsilon_{s}}\right| /(\pi \hbar)$. The functions $\Gamma\left(\mathbf{k}^{\prime}, \mathbf{k}\right)$ and $\Omega\left(\mathbf{k}^{\prime}, \mathbf{k}\right)$ depend only on the radial variables $k$ and $k^{\prime}$ and are denoted by $\Gamma_{k^{\prime}, k}$ and $\Omega_{k^{\prime}, k}$, respectively where

$$
\Gamma_{k}= \begin{cases}c_{2} \ln \left(\left(k+\sqrt{k^{2}-\omega_{1}}\right) / \sqrt{\omega_{1}}\right) / k, & \text { if } k^{2} \geq \omega_{1} \\ 0, & \text { if } k^{2}<\omega_{1},\end{cases}
$$

with $\omega_{1}=2 m \omega_{q} / \hbar, \quad c_{2}=\left(m e^{2} \omega_{q} / \hbar^{2}\right)\left|1 / \epsilon_{\infty}-1 / \epsilon_{s}\right|$. The Neumann series corresponding to equation (3) converges [3] and a $\mathrm{MC}$ approach can be applied to evaluate the electron energy distribution. We note that this approach can be generalized for finite temperatures in a straightforward way.

\section{Monte Carlo Approach}

Define a terminated Markov chain $\left(\kappa_{0}, \tau_{0}\right) \rightarrow \ldots \rightarrow\left(\kappa_{j}, \tau_{j}\right) \rightarrow \ldots \rightarrow\left(\kappa_{l_{\varepsilon}}, \tau_{l_{\varepsilon}}\right)$, such that every point $\left(\kappa_{j}, \tau_{j}\right) \in(0, Q) \times\left(0, \tau_{j-1}\right), \quad j=1,2, \ldots, l_{\varepsilon}$ ( $\varepsilon$ is the truncation parameter) is sampled using an arbitrary transition density function $r\left(k, k^{\prime}, t, t^{\prime \prime}\right)$ which is tolerant1 to both kernels in equation (3).

The biased Monte Carlo estimator for the solution of equation (3) at the fixed point $k=\kappa_{0}$ at the time $t=\tau_{0}$ using backward time evolution of the numerical trajectories has the following form:

\footnotetext{
$1 r(x)$ is tolerant of $g(x)$ if $r(x)>0$ when $g(x) \neq 0$ and $r(x) \geq 0$ when $g(x)=0$.
} 


$$
\begin{gathered}
\xi_{l_{\varepsilon}}\left[\kappa_{0}, \tau_{0}\right]=\phi\left(\kappa_{0}\right)+\sum_{j=1}^{l_{\varepsilon}} W_{j}^{\alpha} \phi_{\alpha}\left(\kappa_{j}\right), \\
W_{j}^{\alpha}=W_{j-1}^{\alpha} \frac{K\left(\kappa_{j-1}, \kappa_{j}\right) K_{\alpha}\left(\kappa_{j-1}, \kappa_{j}, \tau_{j-1}, \tau_{j}\right)}{p_{\alpha} r\left(\kappa_{j-1}, \kappa_{j}, \tau_{j-1}, \tau_{j}\right)}, W_{1}^{\alpha}=1, \alpha=1,2, j=0, \ldots, l_{\varepsilon} .
\end{gathered}
$$

The probabilities $p_{\alpha}(\alpha=1,2)$ are related to the choice of one of the kernels. Now we can define a Monte Carlo method

$$
\frac{1}{N} \sum_{i=1}^{N}\left(\xi_{l_{\varepsilon}}\left[\kappa_{0}, \tau_{0}\right]\right)_{i} \stackrel{P}{\longrightarrow} f\left(\kappa_{0}, \tau_{0}\right)
$$

where $\left.\left.\left.\xi_{l_{\varepsilon}}\left[\kappa_{0}, \tau_{0}\right]\right)_{1}, \xi_{l_{\varepsilon}}\left[\kappa_{0}, \tau_{0}\right]\right)_{2}, \ldots, \xi_{l_{\varepsilon}}\left[\kappa_{0}, \tau_{0}\right]\right)_{N}$ are independent values of the estimator (4) and $\stackrel{P}{\longrightarrow}$ means stochastic convergence as $N \rightarrow \infty$. The relation (5) still does not determine the computation algorithm: we must specify the modeling function (sampling rule) $\xi_{l_{\varepsilon}}\left[\kappa_{0}, \tau_{0}\right]=g\left(\beta_{1}, \ldots, \beta_{n}\right)$, where $\beta_{1}, \ldots, \beta_{n}$ are uniformly distributed random numbers in the interval $(0,1)$. Now both relations (5)) and the sampling rule define a Monte Carlo algorithm for (41).

Thus we can say [4] the constructive dimension (c.d.) of the algorithm is $n$, i.e. $\quad c . d .=n$. Clearly, the variance of the MC estimator (4) does not depend on the c.d. Nevertheless, the c.d. has suggested a classification of sampling rules and an ordering of tests for pseudo-random numbers.

The transition density function in the Markov chain can be chosen in the following way $r_{\alpha}\left(k, t, k^{\prime}, t^{\prime \prime}\right)=r\left(k, k^{\prime}\right) r\left(t, t^{\prime \prime} / k, k^{\prime}\right), \quad \alpha=1,2$, where

$$
r\left(k, k^{\prime}\right)=\bar{C} \frac{k^{\prime}}{k} \ln \left(\frac{k+k^{\prime}}{\left|k-k^{\prime}\right|}\right) \text { and } r\left(t, t^{\prime \prime} / k, k^{\prime}\right)=\frac{\Gamma_{k, k^{\prime}} \exp \left(-\Gamma_{k, k^{\prime}}\left(t-t^{\prime \prime}\right)\right)}{1-\exp \left(-\Gamma_{k, k^{\prime}} t\right)} \text {. }
$$

The normalized density function $r\left(k, k^{\prime}\right)$ can be expressed as an infinite weighted sum of other density functions by expanding $\left(k^{\prime} / k\right) \ln \left(\left(k+k^{\prime}\right) /\left(\left|k-k^{\prime}\right|\right)\right)$, i.e.

$$
\begin{gathered}
r\left(k, k^{\prime}\right)=\sum_{i=0}^{\infty} \bar{C}_{i} r_{i}\left(k, k^{\prime}\right), \quad \bar{C}_{i} \geq 0, \quad \sum_{i=0}^{\infty} \bar{C}_{i}=1, \\
r_{i}\left(k, k^{\prime}\right)= \begin{cases}(2 i+3) \frac{\left(k^{\prime}\right)^{2 i+2}}{k^{2 i+3}}, & \text { when } 0 \leq k^{\prime}<k \\
(2 i-1)\left[\frac{(Q k)^{2 i-1}}{Q^{2 i-1}-k^{2 i-1}} \frac{1}{\left(k^{\prime}\right)^{2 i}}\right], & \text { when } k<k^{\prime} \leq Q,\end{cases} \\
\bar{C}_{i}= \begin{cases}\frac{2}{(2 i+1)(2 i+3)}, & \text { when } 0 \leq k^{\prime}<k \\
\frac{4 k^{2}}{\left(4 i^{2}-1\right)}\left(1-\left(\frac{k}{Q}\right)^{2 i-1}\right) & \text { when } k<k^{\prime} \leq Q .\end{cases}
\end{gathered}
$$

The decomposition MC approach can be applied to sample $k^{\prime}: 1$. Generate $\beta_{1}, \beta_{2}, \beta_{3}$ uniform on $[0,1] ; 2$. Define $\bar{C}_{i}$ by $\beta_{1}$ using decomposition MC techniques. 3. Sample $k^{\prime}$ with the $i$-th density function $r_{i}\left(k, k^{\prime}\right)$, namely, $k^{\prime}=k\left(\beta_{3}\right)^{\frac{1}{2 i+1}}$, if $\beta_{2} Q<k$. Otherwise, $k^{\prime}=k /\left[1-\beta_{3}\left(1-(k / Q)^{2 i-1}\right)\right]^{\frac{1}{2 i-1}}$.

Using the normalized conditional probability density function $r\left(t, t^{\prime \prime} / k, k^{\prime}\right)$ we can sample $t^{\prime \prime}=\log \left(\beta_{4}\left(\exp \left(\Gamma_{k, k^{\prime}} t\right)-1\right)+1\right) / \Gamma_{k, k^{\prime}}$, where $\beta_{4} \in(0,1)$. Finally, we 
generate $\beta_{5} \in(0,1)$ and choose one of the kernels $K_{\alpha}\left(k, k^{\prime}, t, t^{\prime \prime}\right), \alpha=1,2$ using probabilities $p_{\alpha}=\left|K_{\alpha}\left(k, k^{\prime}, t, t^{\prime \prime}\right)\right| /\left(\left|K_{1}\left(k, k^{\prime}, t, t^{\prime \prime}\right)\right|+\left|K_{2}\left(k, k^{\prime}, t, t^{\prime \prime}\right)\right|\right)$. Summarizing, we have used 5 uniform random numbers $\beta_{1}, \ldots, \beta_{5}$ in order to construct the MC estimator (4) for one transition $(k, t) \rightarrow\left(k^{\prime}, t^{\prime \prime}\right)$ in the Markov chain.

The computational complexity of the obtained iterative MC algorithm can be measured by the quantity $F=N \times t_{n_{0}} \times E\left(l_{\varepsilon}\right)$. We note that the number of the random walks, $N$, and the average number of transitions in the Markov chain, $E\left(l_{\varepsilon}\right)$, are connected with the stochastic and systematic errors [3]. However the mean time for modeling one transition, $t_{n_{0}},\left(n_{0}=5\right)$ depends on the complexity of the transition density functions and the choice of the random number generator. It is strongly recommended that all simulations be done with two or more different generators, and the results compared to check whether the prng is introducing a bias. The c.d. of this algorithm can be defined as the average number of uniformly distributed random numbers necessary for carrying out one trial, i.e. c.d. $=n_{0} E\left(l_{\varepsilon}\right)$. Thus we can use parallel prng's that produce $n_{0}=5$ independent and non-overlapping random sequences in order to compute every transition in the Markov chain as well as sampling 5 consecutive pseudo-random numbers from a sequential generator.

\section{Numerical Results and Discussions}

The simulation results are obtained for GaAs with material parameters taken from [2]. The initial condition is a Gaussian function of the energy. The solution $f(k, t)$ is estimated by the MC estimator in 60 points of the simulation domain between 0 and $Q=66 \times 10^{7} / \mathrm{m}$.

The iterative MC algorithm is realized using the following sequential prng's: 1. CLCG-PL, Combined linear congruential generator with parameters recommended by P. L'Ecuyer [5]; 2. EICG, Explicit inversive congruential generator 6]; 3. ICG, Inversive congruential generator 7]; 4. LCG-F, Linear congruential generator with parameters recommended by Fishman [8]; 5. LCG-PM, Linear congruential generator with parameters recommended by Park and Miller [9]; 6. MT-MN, Mersenne Twister generator by Matsumoto and Nishimura 10]; as well as the following parallel prng's: 1. SNWS, Shuffled nested Weyl sequences [1] with a multiplier $M=1234567$. To produce 5 random sequences we use the following seeds: $\gamma=\left\{2^{1 / 2}\right\},\left\{3^{1 / 2}\right\},\left\{5^{1 / 2}\right\},\left\{7^{1 / 2}\right\}$ and $\left\{11^{1 / 2}\right\}$. 2. SPRNG, the Scalable Parallel Random Number Generator Library [12.

The MC algorithm were implemented in the $\mathrm{C}$ language. Numerical tests were performed on a Sun Ultra Enterprise 450 with 4 Ultra-SPARC, $400 \mathrm{MHz}$ CPUs running Solaris. In all our tests $\varepsilon=0.0001$. Such a choice of the truncation parameter allow us to ignore the systematic error [3] and to investigate whether any generator under consideration is introducing a bias when different stochastic errors are fixed. The quantity presented on the $y$-axes in all figures below, $k f(k, t)$, is proportional to the electron energy distribution function multiplied by the density of states. The quantity $k^{2}$ given on the $x$-axes in units of $10^{14} / \mathrm{m}^{2}$ is proportional to the electron energy. Figure 1 compares the solutions for evolu- 
Table 1. Comparison of the accuracy of the solution obtained with the SNWS and SPRNG generators for the 3 points with the biggest variance. The evolution time is $100 \mathrm{fs}$ in the case $N=1$ million $(\mathrm{mln}), 150 \mathrm{fs}$ in the case $N=5$ million $(\mathrm{mln})$ and $200 \mathrm{fs}$ in the case $N=10$ million (mln), respectively.

\begin{tabular}{|c|c|c|c|c|c|c|}
\hline \multirow[b]{2}{*}{$\mathrm{N}$} & \multirow[b]{2}{*}{$k$} & \multicolumn{2}{|c|}{ SNWS } & \multicolumn{2}{|c|}{ SPRNG } & \multirow[b]{2}{*}{$\left|f_{1}-f_{2}\right|$} \\
\hline & & $k f_{1}$ & $\mu$ & $k f_{2}$ & $\mu$ & \\
\hline \multirow{3}{*}{$\begin{array}{c}1 \\
\mathrm{mln}\end{array}$} & 48.00 & \multicolumn{5}{|c|}{$9.8192 \pm 0.0038 \quad 9.7896 \pm 0.0038 \quad 6.2 \times 10^{-4}$} \\
\hline & 48. & \multirow{2}{*}{\multicolumn{2}{|c|}{$\begin{array}{l}10.4557 \pm 0.0039 \\
10.7340 \pm 0.0039\end{array}$}} & 10.4595 & & \multirow{2}{*}{$\begin{array}{l}7.8 \times 10^{-5} \\
6.4 \times 10^{-4}\end{array}$} \\
\hline & 149. & & & 10.70 & & \\
\hline \multirow{3}{*}{$\begin{array}{c}5 \\
\mathrm{mln}\end{array}$} & 48. & \multicolumn{2}{|c|}{$14.6451 \pm 0.0041$} & 14.702 & 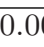 & $1.2 \times 10^{-3}$ \\
\hline & 48. & \multicolumn{5}{|c|}{$15.6877 \pm 0.004315 .7370 \pm 0.00431 .0 \times 10^{-3}$} \\
\hline & 49. & \multirow{2}{*}{\multicolumn{2}{|c|}{$\frac{15.4964 \pm 0.0042}{17.1504 \pm 0.0066}$}} & 15.539 & & $8.7 \times 10^{-4}$ \\
\hline $\operatorname{mln}$ & 48. & & & 16.963 & & $3.9 \times 10^{-3}$ \\
\hline \multirow{2}{*}{10} & 48. & \multicolumn{2}{|c|}{$18.2430 \pm 0.0066$} & 18.05 & & $10^{-3}$ \\
\hline & & 17.7436 & 00 & -7 & & $10^{-3}$ \\
\hline
\end{tabular}

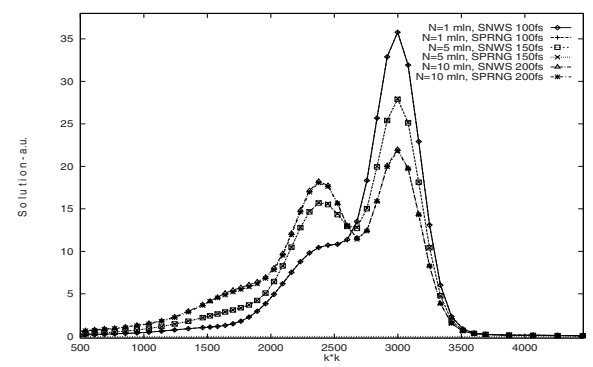

Fig. 1. Comparison of the electron energy distribution $k f(k, t)$ versus $k^{2}$ obtained by using of SNWS and SPRNG.

Table 2. Comparison of the computational complexity of the algorithm using the sequential and parallel prng's.

\begin{tabular}{|c|c|c|c|c|}
\hline \multirow[b]{2}{*}{ generator } & \multicolumn{4}{|c|}{$150 \mathrm{fs}, N=150000 \quad 200 \mathrm{fs}, N=750000$} \\
\hline & $C P U$ time & $E\left(l_{\varepsilon}\right)$ & $C P U$ time & $E\left(l_{\varepsilon}\right)$ \\
\hline MT- & $15 n$ & & $1 \mathrm{~h} 20 \mathrm{r}$ & \\
\hline & 20 & 15.5 & ih & 055 \\
\hline & 23 & & $2 \mathrm{~h}$ & \\
\hline & 23 & 00 & & 55 \\
\hline & 24 & & $2 \mathrm{~h}$ & \\
\hline & $30 n$ & 15 & $2 \mathrm{~h}$ & 99 \\
\hline & 30 & & & 91 \\
\hline CG & $47 \mathrm{~m} 31.30 \mathrm{~s}$ & 15.5153 & $3 \mathrm{~h} 2 \mathrm{~m} 44.73 \mathrm{~s}$ & 15.9023 \\
\hline
\end{tabular}

tion times 100 femtoseconds $(f s), 150 f s$ and $200 f s$ obtained by using the SNWS and, SPRNG parallel prng's. The number of realizations of the MC estimator (41) are 1 million $(\mathrm{mln}), 5 \mathrm{mln}$ and $10 \mathrm{mln}$, respectively. We see that the solutions coincide. Table 1 shows the mean square error, $\mu$, and the absolute error for the 
3 values of the momentum $k$ with the biggest variance using the SNWS and SPRNG generators. In this "the worst" case of the variance compared with the
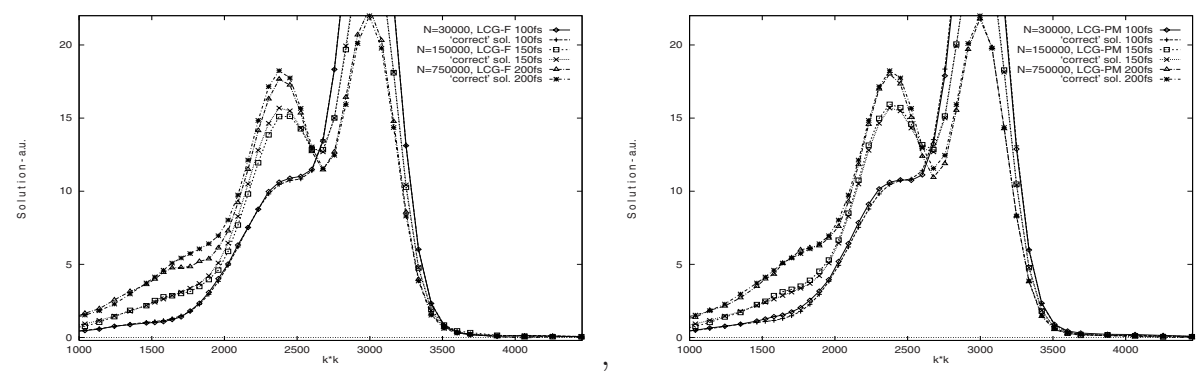

Fig. 2. Comparison of the electron energy distribution obtained by using of LCG-F and LCG-PM generators with the "correct" solution on the left and the right pictures, respectively.

variance at the other points, we have $\mu=O\left(10^{-3}\right)$ and absolute errors are in agreement with the mean square error. Let us note that the exact solution of the B-F equation is unknown. Given the excellent agreement and similar variances, we can take any MC solution from Fig. 1 as a "correct" solution.

Figures 2-5 compare "correct" solutions (using the results with the SNWS generator) for the evolution times $100 \mathrm{fs}, 150 \mathrm{fs}$ and $200 \mathrm{fs}$ with the quantum solutions obtained using all the sequential and parallel prng's when the mean square error is $O\left(10^{-2}\right)$. The number of realizations of the MC estimator are $N=30000,150000$ and 750000. Results obtained when $k^{2}<2000$ for $k f(k, t)$ using the sequential generators when compared with the "correct" solution show systematic differences. The best case occurs when using the CLG-PM, minimal standard, generator. However, for all times it exhibits small consistent differences. Systematic differences in the MC solution with increasing evolution time appear when LCG-F, ICG, EICG and MT-MN are used. Random "noise" in the MC solution is observed when the CLCG-PL generator is used, which, however, is unbiased. When $k^{2}>2000$ the results using any prng's disagree in the first peak of the distribution. This can be explained because the product $k f(k, t)$ for bigger values of $k$ is sensitive to even small errors in the MC solution. Table 2 shows the computational complexity ( $C P U$ time for all 60 points) of the algorithm using all the prng's. We see that the computational cost is the least when the MT-MN generator is used. The CPU time of the algorithm using the SNWS and SPRNG parallel prng's is closely to the CLCG-PL sequential generator and they are faster than the EICG and LCG-F sequential generators. The ICG generator is twiceas slow as the parallel generators with the MC algorithm and therefore it should not be employed for solving this problem. Also, the quantity $E\left(l_{\varepsilon}\right)$ very slowly increases with increasing evolution time. 

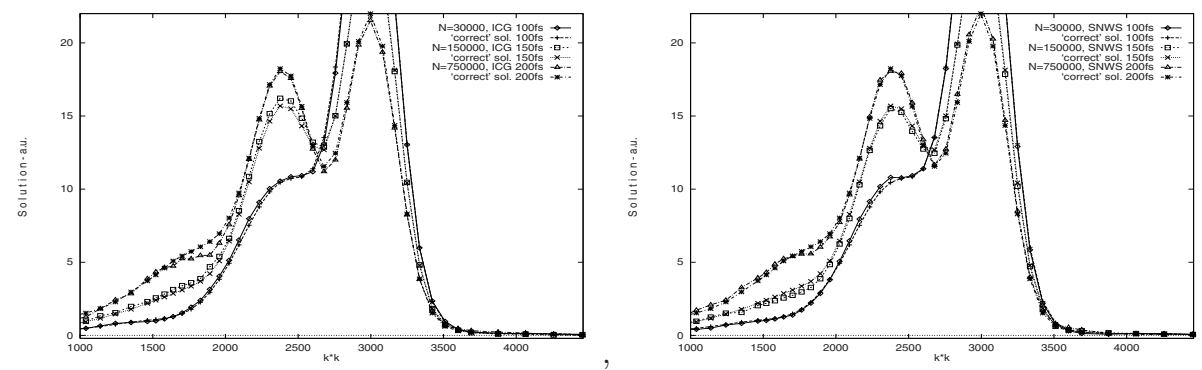

Fig. 3. Comparison of the electron energy distribution obtained by using of ICG and SNWS generators with the "correct" solution on the left and the right pictures, respectively.
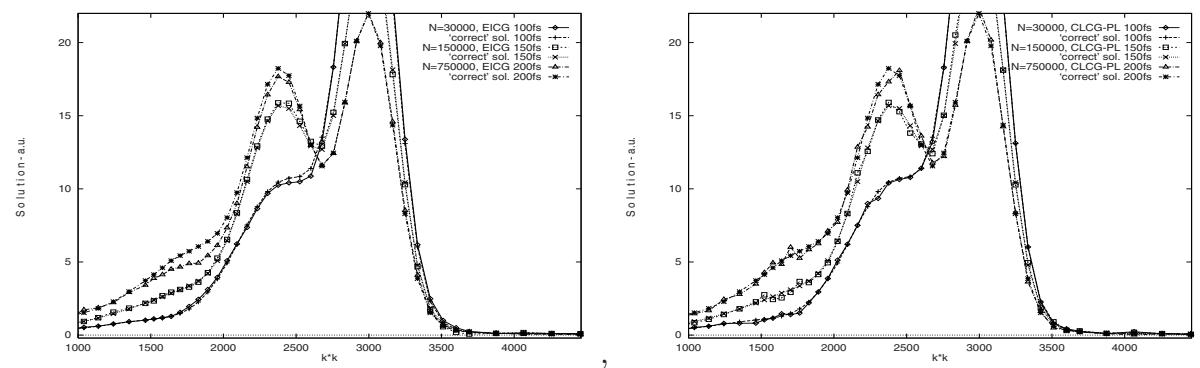

Fig. 4. Comparison of the electron energy distribution obtained by using of EICG and CLCG-PL generators with the "correct" solution on the left and the right pictures, respectively.

\section{Summary}

Statistically, the solution of the B-F equation would be expected to be noisier at $O\left(10^{-2}\right)$ than at $O\left(10^{-3}\right)$ mean square error. It is gratifying that the two parallel prng's used gave the same answer at $O\left(10^{-3}\right)$ precision. However, even at $O\left(10^{-2}\right)$ mean square error, if the solution was unbiased, we would expect random fluctuations about the more precise solution. This was only observed with the CLCG-PL prng. All the other sequential generators exhibited systematic rather than random differences.

Therefore we conclude that parallel prng's are preferable to solve this problem as the evolution time increases. In this case, the $C P U$ time of the algorithm become crucial. Thus, to predict the solution we need parallel realizations of the algorithm and/or we have to estimate the solution with coarser stochastic error. To obtain a high parallel efficiency in the case of the parallel realization of the algorithm, the random sequences have to be produced with similar $C P U$ times. 

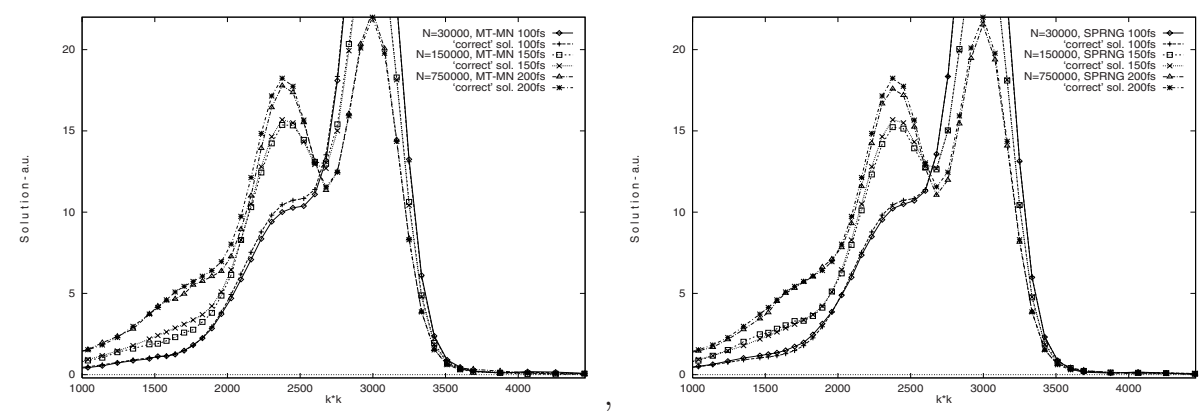

Fig. 5. Comparison of the electron energy distribution obtained by using of MT-MN and SPRNG generators with the "correct" solution on the left and the right pictures, respectively.

\section{References}

1. Barker, J., Ferry, D.: Self-scattering path-variable formulation of high field timedependent quantum kinetic equations for semiconductor transport in the finitecollision-duration regime, Phys. Rev. Lett. 42(26) (1979) 1779-1781.

2. Nedjalkov, M. et al: Statistical modeling of pulse excited electron quantum kinetics in a one-band semiconductor, Math.\& Comp. in Simul. 47 (1998) 391-402.

3. Gurov, T.V., Whitlock, P.A.: An efficient backward Monte Carlo estimator for solving of a quantum kinetic equation with memory kernel, Math. Es Comp. in Simul. 60 (2002) 85-105.

4. Sobol, I.M.: On quasi-Monte Carlo integration, Math.\& Comp. in Simul. 47 (1998) 103-112.

5. L'Ecuyer, P.: Efficient and Portable Combined Random Number Generators, Communications of the ACM 31 (1988) 742-774.

6. Eichenauer-Hermann, J.: Statistical independence of a new class of inversive congruential pseudorandom numbers, Math. Comp. 60 (1993) 375-384.

7. Eichenauer, J., Lehn, J.: A non-linear congruential pseudo-random number generator, Stat. Papers 27 (1986) 315-326.

8. Fishman, G.S.: Multiplicative congruential random number generators with modulus $2^{\beta}$ : an exhaustive analysis for $\beta=32$ and a partial analysis for $\beta=48$, Math. Comp. 54 (1990) 331-344.

9. Park, S.K., Miller, K.W.: Random Number Generators: Good Ones Are Hard to Find, Communications of the ACM 31 (10) (1988) 1192-1201.

10. Matsumoto, M., Nishimura, T.: Mersenne Twister: A 623-Dimensionally Equidistributed Uniform Pseudo-Random Number Generator, ACM Trans. on Modeling E Comp. Simul. 8 (1) (1998) 3-30.

11. Holian,B.L. et al: Pseudorandom number generator for massively parallel molecular-dynamics simulation, Phys. Rev. E 52(2) (1994) 1607-1615.

12. Scalable Parallel Random Number Generators Library for Parallel Monte Carlo Computations, SPRNG 1.0 and SPRNG 2.0 - http://sprng.cs.fsu.edu. 